\title{
Editor's Announcement
}

Mater. Trans. 57 (2016) 99-102

\section{Simulation of Effect of Loading Rate on Compression Properties}

in the Two-Dimensional Model of Aluminum Foam Sandwich Panels

Renjun Dou, Sawei Qiu and Yan Ju

This paper has been retracted from Mater. Trans. by the authors' request due to the following reason.

Firstly, we made a preliminary estimate on the density $\left(\rho_{0}\right)$ of AFS panels before the plastic wave because we did not fully understand the property of AFS panels, which made the calculation results according to the value of the density $\left(\rho_{0}\right)$ of AFS panels, that was wrong in the paper. For example, the locking density $\left(\rho_{1}\right)$ of AFS panels with relative density 0.3 in Table 2 were incorrect, according to the following formula:

$$
\rho_{1}=\frac{\rho_{0}}{1-\varepsilon_{1}}
$$

Table 2 Calculation results of AFS panels with relative density 0.3.

\begin{tabular}{llll}
\hline$v_{1} / \mathrm{m} \cdot \mathrm{s}^{-1}$ & $v_{p} / \mathrm{m} \cdot \mathrm{s}^{-1}$ & $\varepsilon_{1}$ & $\rho_{1} / \mathrm{kg} \cdot \mathrm{m}^{-3}$ \\
\hline 80 & 129.03 & 0.62 & 2131.59 \\
160 & 238.81 & 0.67 & 2454.55 \\
240 & 342.86 & 0.70 & 2700 \\
\hline
\end{tabular}

Especially the further research results (unpublished) in the subsequent study have conflict with the above-mentioned results in the work. Secondly, with the increase of loading rate, the stress of bottom of AFS panels increases in the process of compression. However, the impact stress of loading surface is only analyzed and it is not accurate for the explanation of the mechanism for the stress of bottom of AFS panels. In addition, under the loading rate $0.01 \mathrm{~m} / \mathrm{s}$, the stress-strain curve of AFS panels are stated in the paper. However, the corresponding mechanism cannot explain the stress-strain curve of AFS panels under the loading rate $0.01 \mathrm{~m} / \mathrm{s}$. All of these could mislead the readers. At last, the withdrawal is meaningful for the preciseness of our work, and it is helpful for the subsequent research of AFS panels. 


\title{
Simulation of Effect of Loading Rate on Compression Properties in the Two-Dimensional Model of Aluminum Foam Sandwich Panels
}

\author{
Renjun Dou*, Sawei Qiu and Yan Ju \\ Faculty of Mechanical and Electrical Engineering, Kunming University of Science and Technology, \\ Jingming South Road, Chenggong University Town, Kunming 650500, Yunnan, China
}

A two-dimensional finite element model of aluminum foam sandwich (AFS) panels was approximated by combing C++ and ANSYS/LSDYNA software to represent the shapes of the cells and geometric distributions. Under different loading rates, the deformation behavior, shock wave propagation process, inertial effect and stress of the bottom of the model of AFS panels are discussed resulting from simulation. We found that plastic deformation in the model first occurs in a weak section in the quasi-static compression simulation, whereas the local densification is obvious during the high-speed impact process. The results also indicate that the speed of the plastic wave, locking density and locking strain increase respectively with increasing loading rate. In addition, under the loading rate $80 \mathrm{~m} / \mathrm{s}$, the model can be compressed after the plastic wave reaches the bottom of the model during the process of deformation due to the reflection of the plastic wave, whereas the plateau stress rises with an increase in the loading rate. [doi:10.2320/matertrans.M2015359]

(Received September 24, 2015; Accepted November 18, 2015; Published January 25, 2016)

Keywords: compression property, aluminum foam sandwich panel, ANSYS/LS-DYNA software, locking density,

\section{Introduction}

Aluminum foam sandwich (AFS) panel is a layered structure, obtained generally by bonding (or welding) two thin but strong and stiff outer skins to a relatively thick and high-porosity aluminum core, whose main advantage is that the existing face sheets improve performance of the structure to bear tensile loads and the surface quality compared with bare foam without face sheets. ${ }^{1)}$ As a relatively new class of materials, AFS panels show good potentials due to their light weight, high strength-to-weight, high stiffness-to-weight, good energy-absorbing capacity, good electromagnetic shielding, and so on, which are widely applied to aerospace, marine, electronics, automotive, construction engineering and other fields. $^{2-4)}$

In recent years, the potentials of AFS panels and other foam materials have driven their researches on the structures and properties in a deep going way, resulting in an extension of the knowledge and related applications. Liu et al. ${ }^{5)}$ reconstructed conyeniently two-dimensional models of aluminum foams from microstructure images and investigated the influences of relative density and strain rate effect on compressive behaviors of aluminum foams. Based on MATLAB and $\mu \mathrm{CT}$ seanning technology, the finite element model (FEM) of aluminum foam core was established and the failure mode of AFS panels is discussed by using ABAQUS/ Explicit software by Zhu et al. ${ }^{6}$ ) The study results showed that damage initiation criterion and linear damage evolution law can describe collapse models of AFS panels under static three-point bending and critical loads of numerical calculation were in accordance with theoretical modeling. Kang et $a .^{7)}$ analyzed the stress enhancement of open cell aluminum foams and pointed out that the variation trends of the stress-time curves of aluminum foams with different cell sizes and relative densities are basically the same and stress enhancement happens in all the materials by the experiment and simulation.

*Corresponding author, E-mail: 1320985749@qq.com
However, as far as we have known, the research on compression behavior of AFS panels under different loading rates is few. Under uniaxial compression experiments, deformation behavior of AFS panels is difficult to be observed thoroughly during the process of high-speed impacting because of the discreteness of experimental results. Therefore, in order to make up for the inadequacy of the experiments, a two-dimensional random model of AFS panels is constructed by combing $\mathrm{C}++$ and ANSYS/LSDYNA software according to uneven distributions of cells of AFS panels. A main purpose of current research is by using the finite element method analyzing the shock wave propagation, the inertial effect and the deformation behavior for AFS panels in the process of compression.

\section{Modeling and Simulating}

Compared with three-dimensional models of AFS panels, two-dimensional random models used in this paper to study the compression behavior of AFS panels have the advantages of the simple geometry shapes and the easy dividing of meshes in ANSYS/LS-DYNA software, and it has been confirmed that two-dimensional random models have a good application in a large number of simulation experiments. ${ }^{8,9)}$ Based on these reasons above, AFS panels are simplified to two-dimensional random models by $\mathrm{C}++$ and ANSYS/LSDYNA software which are composed of different size of the sphere holes so that achieving a better simulation effect of AFS panels in this work. The model of AFS panels is based on a highly porous aluminum foam core $(20 \mathrm{~mm}$ width, $20 \mathrm{~mm}$ thickness) and two face sheets $(20 \mathrm{~mm}$ width, $0.8 \mathrm{~mm}$ thickness) which are adhered to two sides of the core, as shown in Fig. 1. The parameters of matrix materials of the model are shown in Table 1. ${ }^{10)}$ The modeling process of AFS panels is illustrated in detail in the previous work. ${ }^{11)}$ In the simulation, relative density 0.3 and loading rates $(0.01 \mathrm{~m} / \mathrm{s}$, $80 \mathrm{~m} / \mathrm{s}, 160 \mathrm{~m} / \mathrm{s}, 240 \mathrm{~m} / \mathrm{s}$ ) are selected, respectively so that simulating quasi-static, medium and high speed compression of AFS panels. AFS panels with larger pore diameters have 


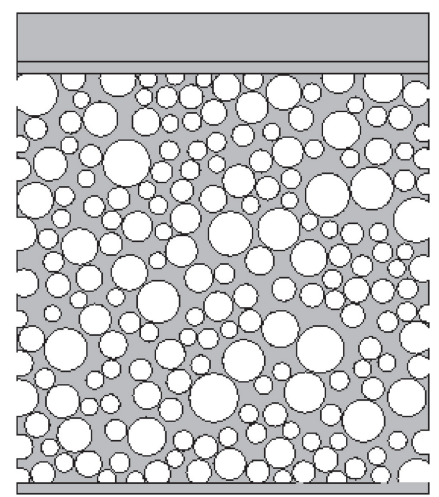

Fig. 1 Two-dimensional random model of AFS panels.

Table 1 Matrix material properties of AFS panels with relative density $0.3 .^{10)}$

\begin{tabular}{ccccc}
\hline $\begin{array}{c}\text { Density, } p / \\
\mathrm{kg} \cdot \mathrm{m}^{-3}\end{array}$ & $\begin{array}{c}\text { Young's } \\
\text { modulus, } E / \\
\mathrm{MPa}\end{array}$ & $\begin{array}{c}\text { Poisson } \\
\text { Ratio, } v / \\
\mathrm{MPa}\end{array}$ & $\begin{array}{c}\text { Yield } \\
\text { strength, } \sigma / \\
\mathrm{MPa}\end{array}$ & $\begin{array}{c}\text { Tangent } \\
\text { modulu, } T / \\
\mathrm{MPa}\end{array}$ \\
\hline 2700 & 70000 & 0.33 & 240 & 10 \\
\hline
\end{tabular}

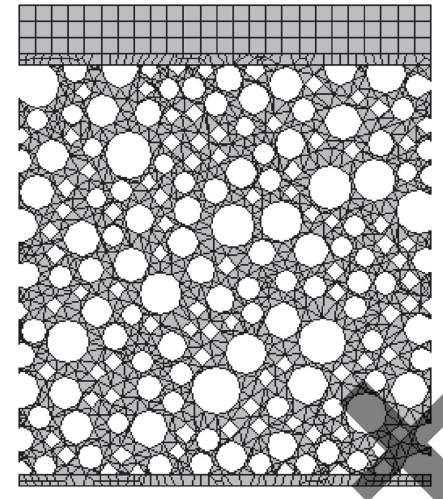

Fig. 2 Mesh generation diagram

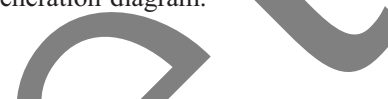

lower yield strengths at the same porosity and loading rate. ${ }^{12)}$ Thus, in order to simulate the practical foam structure and the influence of loading rate in the paper, the cells distribute randomly in foam structure and the pore diameter of the models varies from 1 to $4 \mathrm{~mm}$. Because of the uneven cell distributions and a large amount of internal cells for aluminum foam core, different extent of local deformations derived from the large strain are generated in the process of compression. Therefore, the constitutive model of AFS panels is set as bilinear kinematic plasticity model to take into account the plastic content and neglect the effect of strain rate for describing the dynamic deformation process of AFS panels. ${ }^{12)}$ To improve the accuracy and speed of analysis, AFS panels are carried on the mesh generation by using the adaptive meshing techniques on account of irregular foam structure, as shown in Fig. 2. The potential contact between cell walls is treated automatically during the compression process by the use of auto-dimensional contact. The displacement is applied to the model from the top under different loading rates by the pressure head and the bottom of the model is limited in all the direction. It can be found that

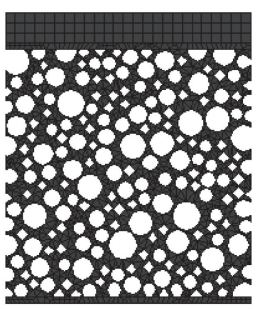

(1)

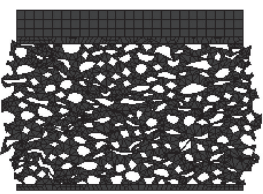

(4)

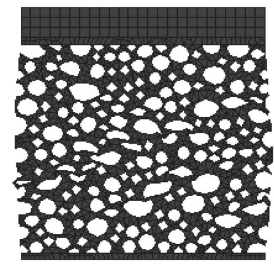

(2)

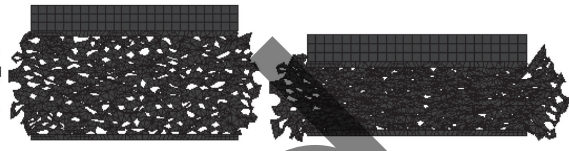

(5)

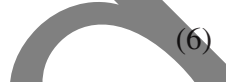

Fig. 3 Deformation behavior of AFS panels with relative density 0.3 under loading rate $0.01 \mathrm{~m} / \mathrm{s}$.

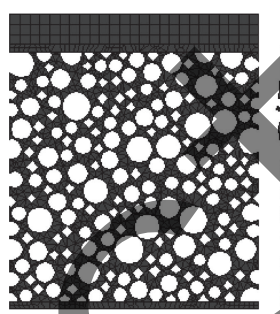

(1)

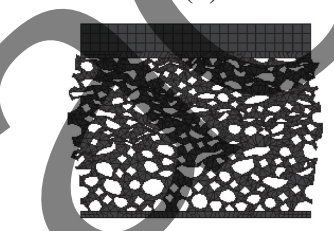

(4)

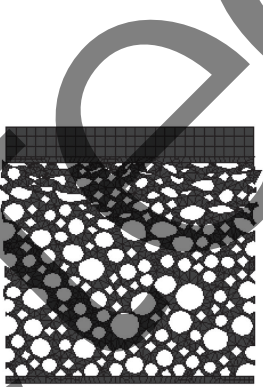

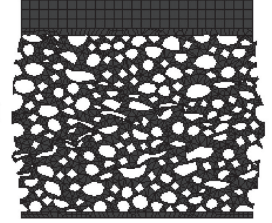

(3)
(2)

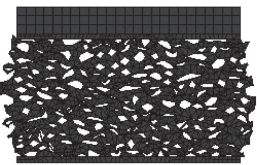

(5)
Fig. 4 Deformation behavior of AFS panels with relative density 0.3 under loading rate $80 \mathrm{~m} / \mathrm{s}$.

the gases in cells can be disregarded which have little effect on deformation of the cells and mechanical property of AFS panels due to low stiffness of gases through the fluidstructure coupling method in the simulation.

\section{Results and Discussions}

\subsection{Deformational behavior of the model}

From Figs. 3-5, it can be observed that compressive deformation process of AFS panels with relative density 0.3 under different loading rates. As shown in Fig. 3, in the uniaxial quasi-static compression process, firstly, middle section produces plenty of plastic distortions and yields since cell walls of large cells have large slenderness ratios and the section is weak to bear force, where irregular deformation bands gradually form on the base of the uneven structure. With the increase of the strain, cell walls gradually contact with each other but compressive capacity increases in the bands, which can lead to other weak cell walls to produce plenty of plastic distortions and yields. When all the cell walls basically contact with each other, AFS panels enter into the densification stage. In the stage, due to massive 


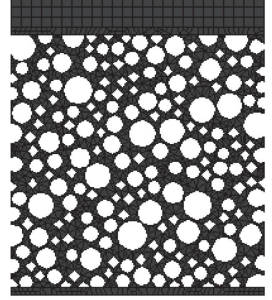

(1)

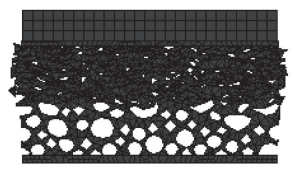

(4)

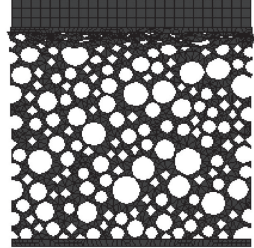

(2)

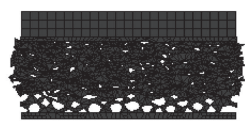

(5)

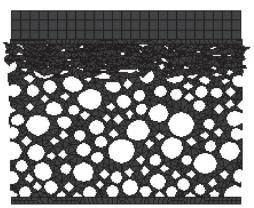

(3)

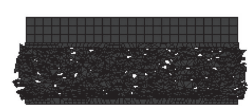

(6)
Fig. 5 Deformation behavior of AFS panels with relative density 0.3 under loading rate $240 \mathrm{~m} / \mathrm{s}$.

reductions of cells, the matrix materials of the model occur plastic flow, which results in the model to produce a large amount of transverse deformations, as shown in Fig. 3. Different from the situation of the uniaxial quasi-static compression, under the loading rate $240 \mathrm{~m} / \mathrm{s}$, the high stress brings about plenty of sharp collapses and local densifications near the stress-bearing surface. The model is approximatively divided into two obvious districts, namely elastic deformation region and plastic deformation region. The plasticity appears and propagates in the loading direction. In order to resist external load for the model of relative density $30 \%$, the cell walls begin to plastic flow in the transverse direction after the effective plastic strain in the loading direction reaches a very high level. Finally, the elastic deformation disappears and the model is basically dense until plastic wave spreads to the bottom. Under the loading rate $80 \mathrm{~m} / \mathrm{s}$, compared with the loading rate $240 \mathrm{~m} / \mathrm{s}$, the plastic wave and the local densification of the model is not prominent, implying that the deformation mode is in the transition stage from quas-static to dynamic state.

\subsection{Nominal stress-strain curves of the model}

As novel shielding materials, the research on anti-impact and energy absorption properties of AFS panels draws extensive attentions of the researchers to rationally use the foam materials. In the following calculations, the influences of loading rate on nominal stress-strain curves of bottom of the model are investigated. Amount of compression along the loading direction and stress of bottom in the model can be obtained by the post-processing module in ANSYS/LSDYNA software. After that, according to the formula (1), the strain $\varepsilon$ can be calculated.

$$
\varepsilon=U_{\mathrm{y} 1} / h
$$

where $U_{\mathrm{y} 1}$ is amount of compression along the loading direction, $h$ is the height of the model. Figure 6 shows the nominal stress-strain curves of bottom of the model under different loading rates. As seen from Fig. 6, at the beginning, initial points of the curves delay to a certain degree and the initial point is far back at a high loading rate. The maximum mechanism lies in that elastic waves do not reach the bottom of the model in time but the foam structure produces a mass

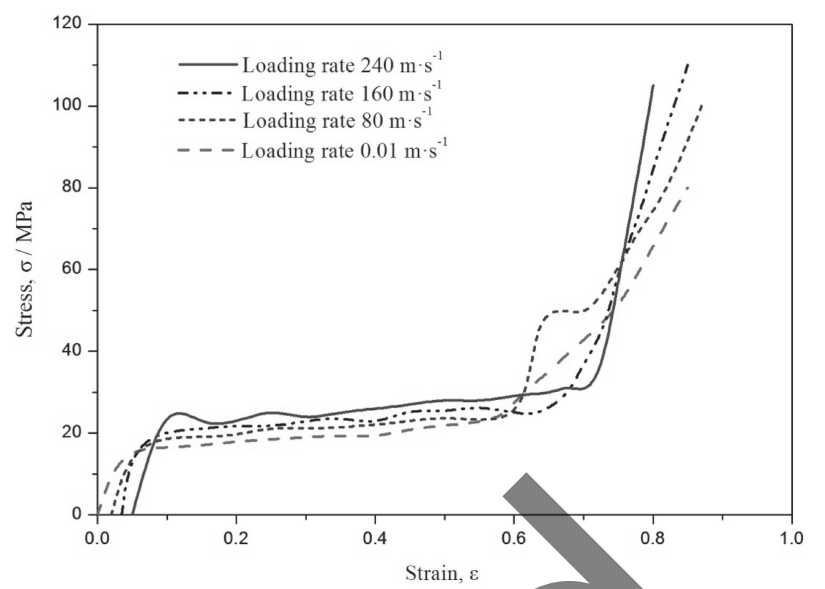

Fig. 6 Nominal stress-strain curves of AFS panels with relative density 0.3 under different loading rates.

of deformations which loading rate in the compression process. It can be seen from Fig. 6, when the nominal strain is about 0.05 , the nominal stress-strain curve begins to rise, meaning that the elastic waves reach the bottom for the model under the loading rate $240 \mathrm{~m} / \mathrm{s}$. From 0.05 to 0.7 , the curve rises slowly because the elastic waves propagate in the model and the cell walls only occurs elastic deformation around the bottom. At the nominal strain 0.7, plastic waves arrival in the bottom but the foam structure is almost dense, so the curve rises sharply.

Under the loading rate $0.01 \mathrm{~m} / \mathrm{s}$, the nominal stress-strain curve has three obvious stages like quasi-static stress-strain curve of AFS panels, namely linear elastic stage, platform stage and densification stage. ${ }^{13)}$

Besides, it can be found the curve has two platform stages and the curve rises again in the strain 0.7 for the model under the loading rate $80 \mathrm{~m} / \mathrm{s}$. As shielding and energy absorption materials, their densification and the re-increase of the stress of bottom of AFS panels should be concerned to guarantee the security of protected objects. As shown in Fig. 4(5), due to inertial effect, the plastic waves reach and strike the bottom of the model at the nominal strain 0.6 , which causes a sharp increase of the stress. In fact, the foam structure is not completely dense, containing a series of cells. These cells produce the deformation and the destruction but provide enough space to be occupied during the second compression, which indicates that the curve can display another platform stage. At the nominal strain 0.7 , the model enters into the densification stage, as shown in Fig. 4(6). All the cells are basically crushed and the curve rises sharply again with the increase of the strain.

\subsection{Propagation law of the stress wave}

It can be directly observed from Figs. 3-5 that the dynamic locking strain, the locking density are larger and the plastic region is more dense under a higher loading rate. From Fig. 6, the locking strain can be directly obtained which is equivalent to the nominal strain when the stress increases sharply in the nominal stress-strain curves. Based on the onedimensional stress wave theory, ${ }^{14)}$ the propagation law of the stress waves of AFS panels is studied under different loading rates. It is assumed that the particles of the model keep 
Table 2 Calculation results of AFS panels with relative density 0.3 .

\begin{tabular}{cccc}
\hline$v_{1} / \mathrm{m} \cdot \mathrm{s}^{-1}$ & $v_{\mathrm{p}} / \mathrm{m} \cdot \mathrm{s}^{-1}$ & $\varepsilon_{1}$ & $\rho_{1} / \mathrm{kg} \cdot \mathrm{m}^{-3}$ \\
\hline 80 & 129.03 & 0.62 & 2131.59 \\
160 & 238.81 & 0.67 & 2454.55 \\
240 & 342.86 & 0.70 & 2700 \\
\hline
\end{tabular}

continuous movements in the process of compression. According to the continuity and physical state of particle movement, the locking density and the speed of the plastic wave can be obtained, respectively, conforming to the following formula:

$$
\begin{gathered}
v_{\mathrm{p}}=\frac{v_{1}}{\varepsilon_{1}} \\
\rho_{1}=\frac{\rho_{0}}{1-\varepsilon_{1}}
\end{gathered}
$$

where $\varepsilon_{1}$ is the locking strain, $\rho_{1}$ is the locking density, $v_{1}$ is the speed of the pressure head, $v_{\mathrm{p}}$ is the speed of the plastic wave and $\rho_{0}$ is the density before the plastic wave. The calculation results under impact loading are shown in Table 2. From Table 2, it can be found that the locking density and the locking strain increase with the increase of the loading rate, which is consistent with observed results above.

Moreover, according to the conversation of energy and physical state of particle before and after waves, the impact stress of the model is as follows:

$$
\sigma_{\mathrm{s}}=\sigma_{\mathrm{y}}+\rho_{0} v_{1}^{2} / \varepsilon_{1}
$$

where $\sigma_{\mathrm{s}}$ is the impact stress of loading surface, $\sigma_{\mathrm{y}}$ is the yield stress of the model. It can be clearly observed that the impact stress is made up of the yield stress, the material density, the locking strain and the loading rate of the model. The latter two items depend on the inertial effect under impact loading. Under dynamic impact loading, the locking strain and the loading rate have a sharp increase as mentioned above, which leads to the impact stress to increase and improves energy absorption capacity of AFS panels. Under loading rate 80 $\mathrm{m} / \mathrm{s}$, the plastic waves can be reflected and the model of the AFS panel again to be compressed after the plastic wave get to the bottom. Therefore, the plateau stress of the bottom during the second compression process is not the impact stress of loading surface. On the basis of the conversation of energy and physical state of particle before and after reflected waves, the plateau stress of the bottom after reflecting can be calculated conforming to the following formula:

$$
\sigma_{\mathrm{p}}=\sigma_{\mathrm{y}}+\rho_{1} v_{1}^{2} /\left(\varepsilon_{2}-\varepsilon_{1}\right)
$$

where $\sigma_{\mathrm{p}}$ is the plateau stress of the bottom of the model after reflecting, $\varepsilon_{2}$ is the locking strain of the second compression. It can be found that the plateau stress has an obvious rise during the second compression increases with the increase of the loading rate because of the inertial effect. When AFS panels are as shielding materials, the stress of protected object will be highly valued. Under the high loading rate, the stress obtained in the bottom of AFS panels during the second compression is considerably larger than their yield stress, which can damage protection objects. In follow-up work, a systematic research will be conducted, combining with protection objects.

\section{Conclusion}

In this paper, a two-dimensional random model of AFS panels is presented and the compression behavior of AFS panels under different loading rates is analyzed by combing $\mathrm{C}++$ and ANSYS/LS-DYNA software. The results indicate that AFS panels have different deformation behaviors under different loading rates. In addition, the loading rate has a significant influence on the speed of the plastic wave, the locking density and the locking strain of AFS panels. Under the medium loading rate $(80 \mathrm{~m} / \mathrm{s})$, AFS panels can occur the second compression, forming a new stress plateau. The study on the compression behavior of AFS panels offers a reliable numerical simulation method, and it is of great significance for the research of AFS panels and making up for the inadequacy of the experiments.

\section{REFERENCES}

Z. Y. Xie, Z. J. Zheng and J. L. Yu: Compos. Part B Eng. 46 (2012) $1-6$

2) A. Pollien, Y. Conde, L. Pambaguian and A. Mortensen: Mater. Sci. Eng A 404 (2005) 9-18.

3) S. Nemat-Nasser, W. J. Kang, J. D. McGee, W.-G. Guo and J. B. Isaacs: Int. J. Impact Eng. 34 (2007) 1119-1146.

4) M. Styles, P. Compston and S. Kalyanasundaram: Compos. Struct. 86 (2008) 227-232.

5) Y. Liu, W. W. Gong and X. Zhang: Comput. Mater. Sci. 91 (2014) $223-$ 230.

6) X. L. Zhu, S. G. Ai, X. F. Lu, K. Cheng, X. Ling, L. X. Zhu and B. Liu: Comput. Mater. Sci. 85 (2014) 38-45.

7) J. X. Kang and L. M. Zhao: J. Vibr. Shock 34 (2015) 189-195.

8) J. Zhang, G. P. Zhao and T. J. Lu: J. Xi'an Jiaotong University 44 (2010) 97-101.

9) H. B. Su, X. Q. Huang and L. Q. Tang: Sci. Technol. Eng. 9 (2009) 6991-6996.

10) Y. H. Yu, Z. Yang and B. Liang: Computer Simulation 27 (2010) $317-$ 341.

11) S. W. Qiu, X. N. Zhang, Q. X. Hao, R. J. Dou, Y. Ju and Y. Hu: Mater. Trans. 56 (2015) 687-690.

12) Z. Chen, Y. J. Liu and G. Z. Kang: J. Science University 44 (2012) 84 87.

13) H. S. Chen, H. Y. Zhao and M. H. Zhang: J. Ningbo University 20 (2007) 118-121.

14) L. L. Wang: Foundations of Stress Waves, (Beijing, National Defense Industry Press, 2005). 